\title{
Hypernatraemic hypovolaemia with anaemia: an unusual presentation of primary testicular insufficiency
}

\author{
Usman Javaid', Vikram Lal1, Catherine Napier', Alison Burbridge ${ }^{2}$ and Richard Quinton ${ }^{1,3}$ \\ 1Department of Endocrinology, Newcastle-upon-Tyne Hospitals, Newcastle upon Tyne, UK, 2Department of \\ Neurorehabilitation, Northumbria, Tyne \& Wear NHS Trust, Newcastle-upon-Tyne, UK, and IInstitute of Genetic \\ Medicine, University of Newcastle-upon-Tyne, Newcastle-upon-Tyne, UK
}

Correspondence

should be addressed

to R Quinton

Email

richard.quinton@ncl.ac.uk

\section{Summary}

Hypogonadal men may experience intense vasomotor symptoms, and vasomotor sweating can occasionally be associated with profound fluid losses. We describe a 37-year-old male, who exhibited persistent hypovolaemic hypernatraemia that was challenging to treat despite a continuous high fluid input ( $>4-5 \mathrm{~L} /$ day). He was noted to have drenching sweats and normochromic anaemia. He had recent traumatic head injury, which resulted in neurocognitive dysfunction, so pituitary function tests were done which showed primary hypogonadism. After exclusion of all other possible causes of excess sweating, hypernatraemia and anaemia, a trial of testosterone therapy was instituted. Sweating dramatically ceased within hours of his first testosterone injection, hydration status normalised within days and anaemia and neurocognitive function progressively improved with continued testosterone replacement. This case demonstrates how, in a susceptible individual, hypovolaemic hypernatraemia can arise from insensible cutaneous fluid loss through eccrine sweating, mediated by vasomotor symptoms of untreated hypogonadism. Although this scenario has not been described in the literature, we felt it needed to be shared with the wider medical community because of how the diagnosis and treatment utterly transformed this patient's functional status and outcome.

\section{Learning points:}

- Hypogonadal men may experience intense vasomotor symptoms and vasomotor sweating can occasionally be associated with profound fluid losses.

- Whether or not there is also hyperosmolar hypernatraemia, clinicians should always consider the possibility of underlying hypogonadism in men with normocytic anaemia and excessive sweating.

- Androgen (testosterone) replacement in hypogonadal men can have a dramatic effect on vasomotor sweating and hot flushes.

\section{Background}

In hospitalised patients, hypernatraemia is a biochemical finding principally associated with hypovolaemia reduced extracellular fluid (ECF) volume - where it is typically associated with raised haemoglobin $(\mathrm{Hb})$ and haematocrit (Hct) due to haemoconcentration effect. However, it may also occur because of pure water loss (e.g. hypodipsia or diabetes insipidus), in which setting it is often associated with normal ECF volume and, rarely, because of hypertonic sodium gain - typically iatrogenic (e.g. resulting from infusion of hypertonic saline or sodium bicarbonate or from hypertonic feeding preparations), in which setting it is often associated with increased ECF 
volume. Hypernatraemia may result in physical and neurocognitive deficits and carries a significant mortality, unless corrected with appropriate fluid resuscitation. Hypernatraemic patients should thus always be evaluated in the light of their ECF volume status, exactly as would be done in the context of hyponatraemia.

The role of intercurrent illness in increasing insensible losses of water via eccrine sweating secretion is often overlooked and is necessarily unrecorded in fluid balance charts except as a fixed $0.5 \mathrm{~L}$ /day assumed loss, but when severe, it has the potential to impact significantly on volume status and electrolytes when patients lack the capacity to increase fluid intake accordingly.

The case outlined above demonstrates how, in a susceptible individual, hypernatraemia can also arise from insensible cutaneous loss through eccrine sweating, mediated by vasomotor symptoms of untreated hypogonadism. Although this scenario has not been described in the literature, we felt it needed to be shared with the wider medical community because of how the diagnosis and treatment utterly transformed a patient's functional status and outcome.

\section{Case presentation}

A 37-year-old man was transferred from a local Neurorehabilitation Unit to the Endocrinology \& Diabetes medical base ward with severe hypernatraemia (Fig. 1), profuse sweating, confusion, intermittent low-grade pyrexia and reduced conscious level, all of which were initially attributed to urosepsis. There was no spontaneous oral fluid intake, but nursing staff and family were able to briefly rouse him sufficiently to drink a cup of water several times a day.

Three months previously, he had sustained significant head trauma, resulting in an extensive subdural haematoma that required evacuation, mechanical ventilation with intracerebro-ventricular pressure monitoring and institution of long-term PEG feeding. His recovery was protracted and his admission characterised by recurrent episodes of pyrexia treated empirically with antibiotics, without any positive cultures. A tendency to hypernatraemia was noted, with biochemical hypovolaemia and very high fluid requirements, averaging $5 \mathrm{~L}$ per day. This typically comprised around $0.3 \mathrm{~L}$ of water

\begin{tabular}{|c|c|c|c|c|c|c|c|c|c|c|c|c|c|c|}
\hline Location: & \multicolumn{4}{|c|}{ Neurosurgical Unit (days 1-56) } & \multirow{7}{*}{ 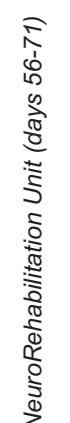 } & \multicolumn{7}{|c|}{ Endocrinology \& Diabetes Medical Base Ward (days 71-132) } & \multicolumn{2}{|c|}{ Endocrine clinics } \\
\hline Days since neurotrauma & 1 & 2 & 30 & 36 & & 71 & 75 & 76 & 106 & 107 & 111 & 125 & 153 & 279 \\
\hline $\mathrm{Hb}(130-180 \mathrm{~g} / \mathrm{L})$ & 136 & 106 & 121 & 119 & & 148 & 111 & 104 & 126 & 119 & 118 & 130 & 142 & 154 \\
\hline Hct $(40-50 \%)$ & 38.9 & 33.6 & 40.7 & 39.1 & & 52.2 & 37.9 & 35.9 & 40.7 & 38.9 & 37.7 & 40.6 & 43.6 & 46.8 \\
\hline $\mathrm{Na}(133-146 \mathrm{mmo} / \mathrm{l})$ & 145 & 146 & 151 & 157 & & 177 & 169 & 157 & 157 & 149 & 145 & 144 & 144 & 144 \\
\hline $\mathbf{K}(3.5-5 \mathrm{mmol} / \mathrm{l})$ & 4.7 & 4.3 & 4.3 & 4.3 & & 3.2 & 3.3 & 4.2 & 4.1 & 3.6 & 4.0 & 4.6 & 4.4 & 4.6 \\
\hline Urea $(2.5-7.8 \mathrm{mmo} / \mathrm{l})$ & 8.7 & 7.9 & 9.0 & 9.7 & & 17.3 & 10.3 & 7.9 & 9.4 & 3.4 & 3.3 & 3.4 & 5.6 & 4.0 \\
\hline Creat $(70-110$ umol/l) & 61 & 64 & 65 & 71 & & 72 & 61 & 47 & 68 & 58 & 58 & 58 & 64 & 69 \\
\hline Urea/Creat ratio (40-100) & 143 & 123 & 138 & 137 & & 240 & 169 & 168 & 138 & 58 & 57 & 58 & 88 & 58 \\
\hline $\begin{array}{l}\text { Osmolality } \\
\text { (274-295 mOsm/kg) }\end{array}$ & & & 310 & & & & & & 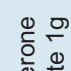 & & & & 둥용 & 品 \\
\hline $\begin{array}{l}\text { urine Osmolality } \\
(\mathrm{mOsm} / \mathrm{kg})\end{array}$ & 704 & & 1132 & & & 1148 & & & 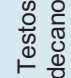 & & & & 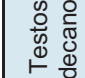 & 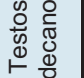 \\
\hline urine $\mathrm{Na}(\mathrm{mmol} / \mathrm{L})$ & & & 175 & & & & & & $=\mathrm{J}$ & & & & & $\xi$ \\
\hline LH (3-13 IU/L) & & & 17.9 & & & & & & & & 14.9 & & 5.3 & 2.1 \\
\hline FSH (1.0-9.2 IU/L) & & & 16.3 & & & & & & & & 11.7 & & 7.1 & 2.3 \\
\hline $\begin{array}{l}\text { Testosterone } \\
(9-25 \mathrm{nmol} / \mathrm{L})\end{array}$ & & & 5.9 & & & & & & & & 18.1 & & 14.4 & 14.5 \\
\hline $\begin{array}{l}\text { free-Testosterone } \\
\text { (215-760 pmol/L) }\end{array}$ & & & 94 & & & & & & & & 286 & & 221 & 250 \\
\hline
\end{tabular}

Figure 1

Serial laboratory parameters over 40 weeks following neurotrauma event. Pink-shaded cells indicate abnormal haematologic parameters, green-shaded cells abnormal biochemical values and blue-shaded boxes sign-post diagnosis and treatment of hypogonadism. 
or juice by mouth; a combination of reduced-sodium enteral feed and water - totalling $2.5 \mathrm{~L}$ - administered via PEG; and around $2.2 \mathrm{~L}$ of intravenous $5 \%$ dextrose. Diabetes insipidus was screened for and unequivocally excluded on the basis of maximally concentrated urine specimens (704 and $1132 \mathrm{mosmol} / \mathrm{kg}$ ). He was transferred from Neuro-ITU to the regional neurorehabilitation unit due to persisting severe neurocognitive deficit and inability to self-care.

Throughout both his inpatient admissions, he exhibited persistent hypovolaemic hypernatraemia (149$177 \mathrm{mmol} / \mathrm{L}$; see green-shaded areas in Fig. 1) that was extremely challenging to treat, with even partial correction of serum sodium was only possible through high daily fluid input (around 5L). This was principally via PEG (feed adjusted by dietician to have the lowest possible salt content), supplemented with intravenous fluids, although he could also be briefly roused to drink oral fluids with sufficient prompting. Clinically, his neurological status remained poor. Verbal communication was almost nonexistent, and he required full assistance with all personal care. Whilst considering potential reasons for the difficulty in managing his hypernatraemia, a pattern of daily, drenching sweating was noted, several times a day, and his skin carried a constant sheen of moisture.

\section{Investigation}

Monitoring of routine haematological parameters revealed persistent normochromic anaemia (see pink-shaded areas in Fig. 1), except for the point of maximum dehydration (day 71) when he was transferred from neurorehabilitation back to the acute hospital. Screens for infectious, inflammatory, metabolic and neoplastic causes of pyrexia were universally negative and two further courses of antibiotics were prescribed without effect. However, review of post head injury endocrine biochemistry (performed to exclude traumatic hypopituitarism) demonstrated unequivocal primary gonadal insufficiency (see blue-shaded lab values in Fig. 1).

Examination with Prader orchidometer revealed atrophic testes (testicular volume 6-8 $\mathrm{mL}$ ) and karyotype was checked (normal 46XY) to exclude Klinefelter's syndrome. A collateral history obtained from the patient's wife and father revealed that excessive sweating had been a longstanding problem. There was a history of unilateral orchidopexy at 5 years of age. Three years previously, the couple had been investigated for primary infertility and given a diagnosis of dual-factor' infertility, based upon his severe astheno- and terato-zoospermia (68\% immotile;
$90 \%$ abnormal), despite a reasonable overall count (38$43 \times 10^{6} / \mathrm{mL}$ ), combined with her low ovarian reserve (AMH $<4.0 \mathrm{pmol} / \mathrm{L}$; satisfactory range: $22-40$ ).

\section{Treatment}

Testosterone replacement therapy had previously been considered and deferred, as it was not considered to be a significant priority in the acute setting. Sir Arthur Conan Doyle's character, Sherlock Holmes, is famous for stating that 'once you eliminate the impossible, whatever remains - no matter how improbable - must be the truth'. Inspired by this logic, and in the absence of any other discernible alternative diagnosis or other means of progressing his care, we hypothesised that (a) insensible fluid loss through eccrine sweating might be of the order of several litres per day and that (b) any intervention that reduced vasomotor symptoms might have a useful effect on hydration status and fluid balance. He therefore received testosterone undecanoate depot (Nebido) $1 \mathrm{~g}$ intramuscularly, to which a dramatic clinical response was rapidly discernible.

\section{Outcome and follow-up}

The drenching, sweating episodes ceased overnight and his skin appeared dry for the first time, with nursing staff no longer having to change his bed-sheets twice-daily. Serum sodium level normalised within forty-eight hours, despite a major reduction in his fluid requirement to under $3 \mathrm{~L} /$ day. Although both haemoglobin and haematocrit showed a brief initial fall due to haemodilution effect, they then rose steadily into the adult male normal range as a result of testosterone-stimulated erythropoeisis.

Crucially, his improved hydration status was accompanied by greatly improved physical and neurocognitive function. In contrast to the minimal verbal communication in the months following his head injury, our patient could now formulate short sentences, sit up out of bed unaided and feed himself independently. This striking change in functional status allowed us to facilitate transfer to the community within 4 weeks. Two months later, he returned to outpatient clinic for administration of a second Nebido injection and further clinical improvement was readily apparent - he was able to rise from his wheelchair, walk a short distance unaided, actively participate in short conversations and write a thank you note to ward nursing staff. He remains on longterm testosterone replacement therapy, but experiences recurrent vasomotor symptoms whenever there is a 
delay in administering his injections beyond an 11-week interval.

\section{Discussion}

The vast majority of people can compensate for insensible fluid losses with adequate fluid intake, driven by their thirst response. Indeed, even some patients with diabetes insipidus choose not to take desmopressin and instead maintain high daily fluid intake and elect to tolerate the inevitable polyuria. Multiple precipitants can result in insufficient volume intake in hospitalised patients, particularly in the context of increased insensible losses. In hospital, hypernatraemia is thus primarily iatrogenic, arising as a result of inadequate or inappropriate prescription of fluids in patients with increased fluid losses, impaired thirst, impaired vasopressin secretion or restricted free water intake (1).

Although we accept that these data do not permit unequivocal assignment of causation, our view is that the most logical and convincing interpretation of the facts underlying this case is of hypovolaemic hypernatraemia arising in a vulnerable patient (a) unable to maintain any spontaneous oral fluid intake and (b) experiencing heightened insensible cutaneous fluid losses through torrential eccrine sweating that overwhelmed the capacity of staff on a busy general ward to adequately replace. The extreme rapidity with which sweating was utterly stopped following testosterone injection, supports our interpretation that it was mediated by the vasomotor symptoms of untreated primary hypogonadism. As this scenario has not yet been described in the literature, we felt that it needed to be shared with the wider medical community because of the manner in which diagnosis and treatment utterly transformed this man's functional and neurocognitive outcome.

Male hypogonadism is a clinical syndrome caused by androgen deficiency, with the potential to adversely affect multiple physiological functions and quality of life. Hypogonadism can be primary (resulting from intrinsic testicular dysfunction), or secondary, due to disruption at the hypothalamic-pituitary-gonadal level. Primary hypogonadism is significantly easier to diagnose due to elevated LH and FSH levels, whereas secondary hypogonadism can be hard to distinguish in the acute setting from non-gonadal illness, because LH, FSH and testosterone levels can be similarly low. Around 1-in-600 men have Klinefelter syndrome (usually 47XXY) and are thus predisposed to develop primary hypogonadism at some point in their lives, and the European Ageing Male
Study found around $2 \%$ of older men to have primary hypogonadism (2).

In the setting of androgen deficiency, subjectively reported symptoms and relevant clinical findings can include asthenia, reduced libido, low mood, impaired cognitive function, impotence and reduced muscle and bone mass. Sweating or 'hot flushes' may also occur in men in the setting of testosterone deficiency, as they do in menopausal females, albeit with significant interindividual variation in severity. Some hypogonadal men and women experience almost no vasomotor symptoms, but others are almost unbearably affected (3).

'Hot flushes' are usually systemic, and therefore, likely to arise after a disruption to the thermoregulatory set point in the hypothalamus (4). The exact cause of this trigger is unknown, but it is likely to initiate mechanisms such as vasodilation (with subsequent flushing and sweating), to elicit a reduction in body temperature (4). Vasomotor sweating can result in marked excursions of insensible water loss and, although estimates of volumes can be difficult to quantify, skin blood flow can reach $7 \mathrm{~L} / \mathrm{min}$ during maximal vasodilation (5). Maximal sweating capacity in a healthy unacclimatised male is around $1.5 \mathrm{~L}$ an hour, but can escalate to $2-3 \mathrm{~L} / \mathrm{h}$ in an acclimatised trained soldier (6). Androgen replacement in hypogonadal men can have a dramatic effect on vasomotor sweating and hot flushes (3).

In our patient, androgen replacement resulted in a rapid reduction in sweating, with a corresponding improvement in hydration status, rapid normalisation in electrolyte parameters, and subsequent normalisation of haematopoiesis. Even in the absence of hyperosmolar hypernatraemia and hyperhydrosis or flushing, clinicians should, in any case, always consider the possibility of underlying hypogonadism in men with otherwise unexplained normocytic anaemia. Properly monitored testosterone replacement therapy for men with a well-founded diagnosis of hypogonadism is a safe, well-tolerated and can transform patient quality of life.

\section{Declaration of interest}

The authors declare that there is no conflict of interest that could be perceived as prejudicing the impartiality of the research reported.

\section{Funding}

This work did not receive any specific grant from any funding agency in the public, commercial, or not-for-profit sector.

\section{Patient consent}

Written informed consent has been obtained. 
Author contribution statement

All authors contributed equally.

\section{References}

1 Palevsky PM, Bhagrath R \& Greenberg A 1996 Hypernatraemia in hospitalised patients. Annals of Internal Medicine 124 197-203. (doi:10.7326/0003-4819-124-2-199601150-00002)

2 Tajar A, Huhtaniemi IT, O’Neill TW, Finn JD, Pye SR, Lee DM, Bartfai G, Boonen S, Casanueva FF, Forti G, et al. 2012 Characteristics of androgen deficiency in late-onset hypogonadism: results from the
European Male Aging Study (EMAS). Journal of Clinical Endocrinology and Metabolism 97 1508-1516.

3 Spetz AE, Fredriksson MG \& Hammar ML 2003 Hot flushes in a male population aged 55,65 , and 75 years, living in the community of Linkoping, Sweden. Menopause: Journal of the North American Menopause Society 10 81-87. (doi:10.1097/00042192-200310010-00013)

4 Stearns V, Ullmer L, López JF, Smith Y, Isaacs C \& Hayes D 2002 Hot flushes. Lancet 360 1851-1861. (doi:10.1016/S0140-6736(02)11774-0)

5 Rowell LB 1986 Human Circulation: Regulation during Physical Strain. New York: Oxford University Press.

6 Wenger CB 1988 Human heat acclimatization. In Human Performance Physiology and Environmental Medicine at Terrestrial Extremes, pp 153-197. Indianapolis, Indiana: Benchmark Press.

Received in final form 3 October 2017

Accepted 11 October 2017 